\title{
OPEN Soil bacterial community as impacted by addition of rice straw and biochar
}

\begin{abstract}
Zhiqiang Tang ${ }^{1,2}$, Liying Zhang ${ }^{2}$, $\mathrm{Na} \mathrm{He}^{2}$, Diankai Gong ${ }^{2}$, Hong Gao², Zuobin Ma², Liang Fu², Mingzhu Zhao ${ }^{2}$, Hui Wang ${ }^{2}$, Changhua Wang ${ }^{2}$, Wenjing Zheng ${ }^{2}$ \& Wenzhong Zhang ${ }^{1 凶}$

The application of straw and biochar can effectively improve soil quality, but whether such application impacts paddy soil bacterial community development remains to be clarified. Herein, the impacts of three different field amendment strategies were assessed including control (CK) treatment, rice straw (RS) application ( $\left.9000 \mathrm{~kg} \mathrm{ha}^{-1}\right)$, and biochar (BC) application $\left(3150 \mathrm{~kg} \mathrm{ha}^{-1}\right)$. Soil samples were collected at five different stages of rice growth, and the bacterial communities therein were characterized via high-throughput 165 rDNA sequencing. The results of these analyses revealed that soil bacterial communities were dominated by three microbial groups (Chloroflexi, Proteobacteria and Acidobacteria). Compared with the CK samples, Chloroflexi, Actinobacteria, Nitrospirae and Gemmatimonadetes levels were dominated phyla in the RS treatment, and Acidobacteria, Actinobacteria, Nitrospirae and Patescibacteria were dominated phyla in the BC treatment. Compared with the RS samples, Chloroflexi, Acidobacteria, Actinobacteria, and Verrucomicrobia levels were increased, however, Proteobacteria, Gemmatimonadetes, Nitrospirae, and Firmicute levels were decreased in the BC samples. Rhizosphere soil bacterial diversity rose significantly following RS and $\mathrm{BC}$ amendment, and principal component analyses confirmed that there were significant differences in soil bacterial community composition among treatment groups when comparing all stages of rice growth other than the ripening stage. Relative to the CK treatment, Gemmatimonadaceae, Sphingomonadaceae, Thiovulaceae, Burkholderiaceae, and Clostridiaceae-1 families were dominant following the RS application, while Thiovulaceae and uncultured-bacterium-o-C0119 were dominant following the BC application. These findings suggest that RS and BC application can improve microbial diversity and richness in paddy rice soil in Northeast China.
\end{abstract}

Paddy rice is a key agricultural crop in China, with japonica rice primarily being cultivated in Northeast China. After harvesting, farmers traditionally burn the rice straw that remains after harvesting before planting rice the following spring. However, air pollution concerns have led to the prohibition of such crop residue burning practices. Instead, agricultural residues are applied to fields in an effort to minimize environmental harm while maximizing resource utilization ${ }^{1}$.

Many researchers have advocated the application of straw and biochar to fields in an effort to improve soil quality and nutrient availability, providing key substrates that can support the growth of root-associated microbes and enzymatic activity ${ }^{2,3}$. Biomass represents a renewable source of fixed carbon (C) that can directly impact soil bacterial community composition and richness relatively quickly ${ }^{4}$. Applying agricultural residues such as rice, wheat, and maize straw can thus significantly improve the $\mathrm{C}$ supply available in a given cropping system.

Biochar (BC) is a solid product derived from the pyrolysis or gasification of crop residues at a low temperature $\left(<500^{\circ} \mathrm{C}\right)$ under anoxic conditions ${ }^{5}$. BC is considered a viable soil amendment compatible with sustainable agricultural practices, and it can significantly improve nutrient availability, soil health, and soil productivity in agricultural contexts ${ }^{6}$. By improving soil water retention and cationic adsorption, $\mathrm{BC}$ can also decrease nutrient loss and improve the quality of acidic soils. $\mathrm{BC}$ application can further slow the release of $\mathrm{C}$ and $\mathrm{N}$ owing to changes in soil properties ${ }^{7}$, thereby influencing microbial community composition and activity ${ }^{8}$. As $\mathrm{BC}$ is porous, it can also serve as an environmental niche compatible with sheltered growth in the soil ${ }^{9}$. Applying BC can thus profoundly alter soil physicochemical properties, thereby impacting microbial richness and diversity. However, the mechanisms whereby $\mathrm{BC}$ alters soil biological properties remain to be fully clarified ${ }^{10}$.

${ }^{1}$ Shenyang Agricultural University, Shenyang, China. ${ }^{2}$ Liaoning Rice Research Institute, Shenyang, China. ${ }^{\square}$ email: zwzhong1@syau.edu.cn 


\begin{tabular}{|l|l|l|l|l|}
\hline Stage & Treatment & Observed OTUs & Chao1 & Shannon \\
\hline \multirow{5}{*}{ Elongation } & RS & 1918 & 1952.2 & 9.64 \\
\cline { 2 - 5 } & BC & 1937 & 1948.1 & 9.52 \\
\cline { 2 - 5 } booting & CK & 1936 & 1951.0 & 9.58 \\
\hline \multirow{5}{*}{ heading } & RS & 1920 & 1933.6 & 9.30 \\
\cline { 2 - 5 } & BC & 1875 & 1920.5 & 9.13 \\
\cline { 2 - 5 } & CK & 1890 & 1909.5 & 9.37 \\
\hline \multirow{5}{*}{ grain filling } & RC & 1923 & 1947.8 & 9.43 \\
\cline { 2 - 5 } & CK & 1933 & 1950.9 & 9.33 \\
\cline { 2 - 5 } & RS & 1941 & 1967.0 & 9.34 \\
\cline { 2 - 5 } & CK & 1823 & 1944.6 & 9.50 \\
\hline \multirow{5}{*}{ ripening } & RS & 1906 & 1945.5 & 9.32 \\
\cline { 2 - 5 } & BC & 1905 & 1936.6 & 9.39 \\
\cline { 2 - 5 } & CK & 1929 & 1939.0 & 9.50 \\
\hline \multirow{2}{*}{} & & & 9.39 \\
\hline
\end{tabular}

Table 1. Estimated operational taxonomic unit (OTU) richness and diversity indexes for soil samples from different stages of rice growth following $16 \mathrm{~S}$ rDNA gene library clustering at 97\% identity after Illumina Hiseq (2500) sequencing. Treatments: $R S$ rice straw applied at $9000 \mathrm{~kg} \mathrm{ha}^{-1}, B C$ biochar applied at $3150 \mathrm{~kg} \mathrm{ha}^{-1}, C K$ soil without straw/biochar application.

Effectively characterizing soil microbial communities is challenging, but recent advances in high-throughput sequencing technologies have significantly aided these efforts ${ }^{11}$. Microbes within the soil can associate with the roots of plants, and modulate crop growth, nutrient uptake, and disease susceptibility ${ }^{12}$. While studies of these microbial communities associated with rice, wheat, and maize plants have shown that both soil type and host genotype influence community composition ${ }^{13,14}$, how these communities respond to BC or straw application remains to be clearly defined. In some studies, soil microbial community composition has been found not to vary when comparing fertilized and non-fertilized soils in a long-term winter wheat, although other studies have instead observed changes in these communities following the application of crop straw or other organic/ inorganic fertilizers. These inconsistent results may be a result of differences in environmental or other management strategies among studies ${ }^{15-17}$.

This study was designed to assess soil bacterial community responses to the application of rice straw (RS) and $\mathrm{BC}$ via a high-throughput $16 \mathrm{~S}$ rDNA sequencing approach. For these analyses, we tracked rhizosphere microbiome development from the elongation to the ripening stage of rice growth in field trials, and we used a Random Forest model to explore the relationships between rhizosphere microbiota composition and rice straw or biochar application.

\section{Results}

Assessment of rice rhizosphere soil bacterial community and richness. In total, over 40,000 valid clean reads were obtained per treatment via a sequence optimization process, after which soil microbial community richness (OTU number) index values were calculated (Table 1). No significant differences in OTUs were observed when comparing samples in different groups (Fig. 1). CK treatment was associated with lower Chaol index values relative to the RS treatment, suggesting that the RS amendment improved bacterial community diversity at the booting stage of rice growth, although community composition tended to stabilize after this stage. Shannon index values in the RS treated samples exhibited improved community diversity relative to samples in the BC or CK treatment groups.

Bacterial community composition. Rhizosphere bacterial community composition during different stages of growth and under different treatment conditions was next assessed (Fig. 2). In comparison with the $\mathrm{CK}, \mathrm{RS}$ and $\mathrm{BC}$ amendment did not alter the top 10 phyla present within the sequenced bacterial community, although significant diversity was observed with respect to the bacteria within each phylum at all five growth stages. The three most dominant phyla in these samples were Chloroflexi, Proteobacteria, and Acidobacteria, with these sequences accounting for $>60 \%$ of total sequences in all soil samples.

Chloroflexi, Actinobacteria, and Gemmatimonadetes levels were higher in RS samples relative to CK samples from the booting stage to the grain filling stage, while Nitrospirae levels were significantly higher relative to CK treatment in all RS-treated samples other than the grain-filling stage samples. RS amendment was associated with a significant increase in Proteobacteria content relative to CK treatment at the ripening stage. BC application was associated with increased Chloroflexi abundance during all growth stages relative to CK treatment. Abundance of Acidobacteria, Actinobacteria, Nitrospirae, and Patescibacteria increased in BC samples relative to CK samples from the booting stage to the grain filling stage.

Over the course of rice growth, the composition of rhizosphere microbial communities changed significantly within each of the three treatment groups (Additional file: Figure S1). In all three treatment groups, Chloroflexi 


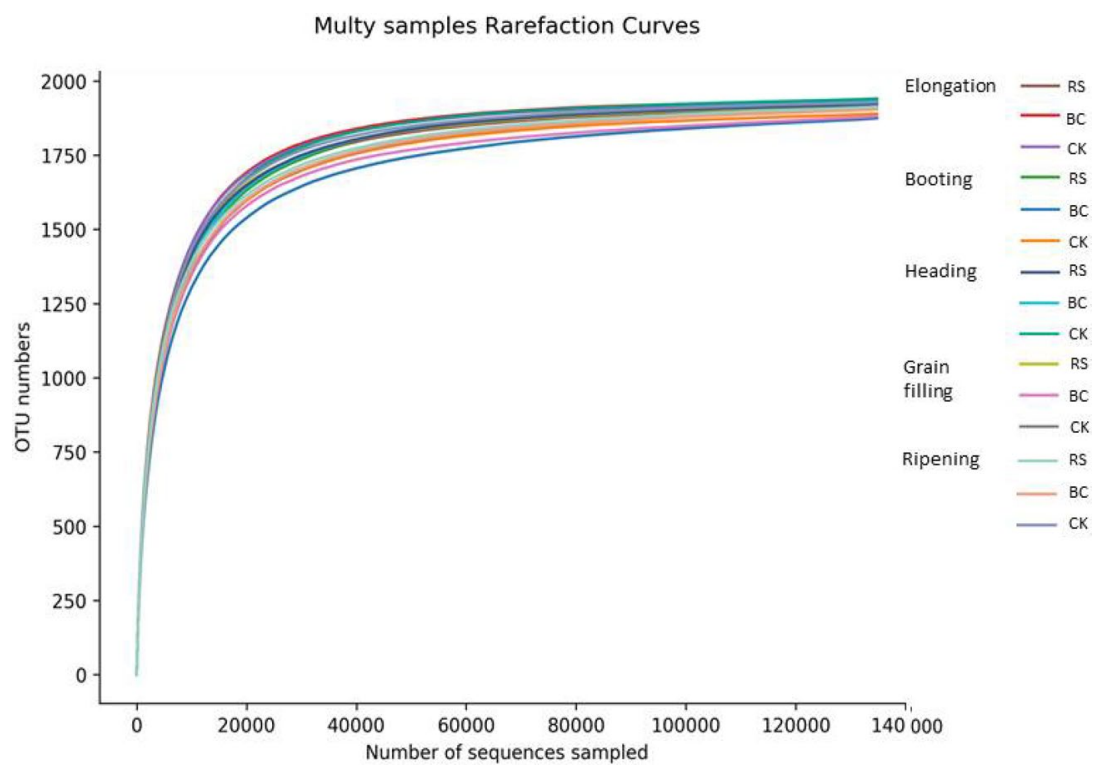

Figure 1. Rarefaction on species-abundance data. Treatments: $R S$ rice straw applied at $9000 \mathrm{~kg} \mathrm{ha}^{-1}, B C$ biochar applied at $3150 \mathrm{~kg} \mathrm{ha}^{-1}$, CK soil without straw/biochar application.

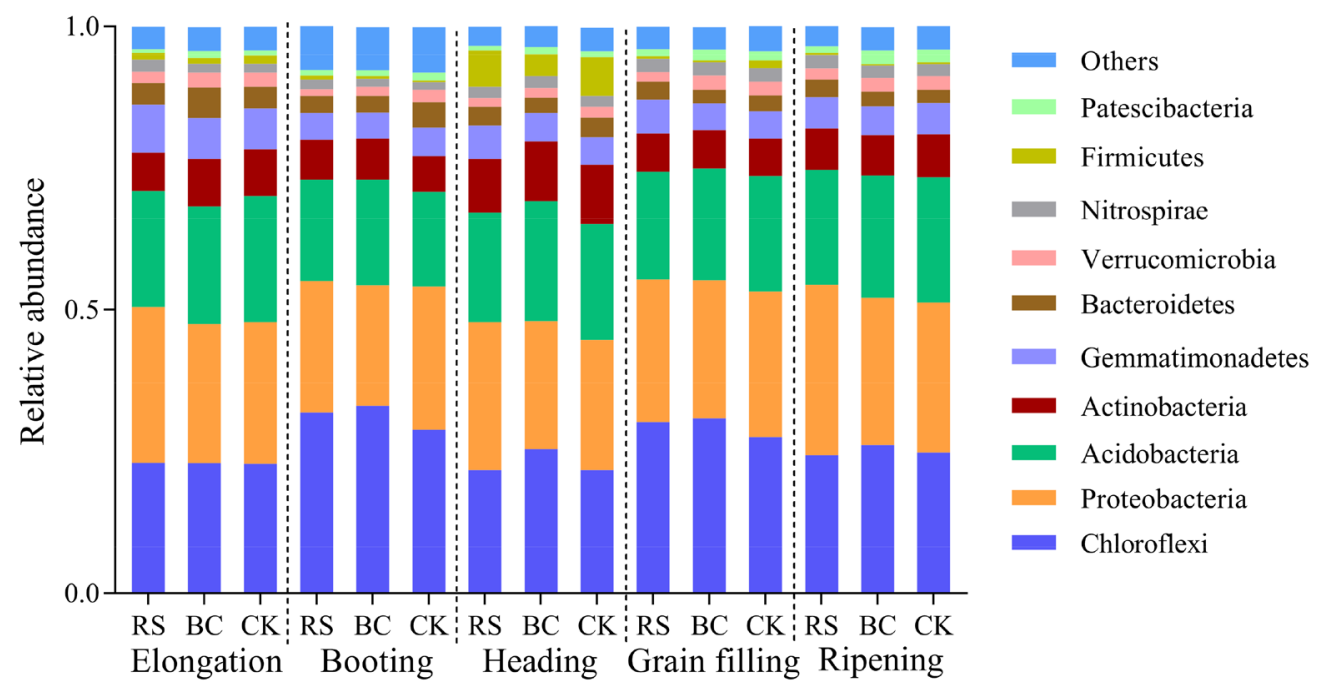

Figure 2. Phylum level bacterial community composition following the rhizosphere treatments. Treatments: RS rice straw applied at $9000 \mathrm{~kg} \mathrm{ha}^{-1}, B C$ biochar applied at $3150 \mathrm{~kg} \mathrm{ha}^{-1}, C K$ soil without straw/biochar application.

abundance was lower at the jointing, heading, and ripening stages of growth, whereas it was elevated during the booting and filling stages. Proteobacteria abundance trended downwards from the elongating to the booting stage, whereas it slowly trended upwards from the booting to the ripening stage in RS-treated (Additional file: Figure S1, RS) and BC-treated (Additional file: Figure S1, BC) samples. In contrast, Proteobacteria abundance in $\mathrm{CK}$ samples remained unchanged from the elongating to the booting stage, declined at the heading stage, and rose at the ripening stage (Additional file: Figure S1, CK). Actinobacteria abundance declined from the elongation to the booting stage, and rose slowly under all treatment conditions. A principal component analysis (PCA) was conducted to further clarify the relationships among microbial communities under these three treatment conditions during the five growth stages. This analysis was conducted using a Mothur calculation approach based upon relative bacterial abundance at the genus level, and the results were visualized using R v.3.0.2 (Fig. 3). In the resultant 2D plot, PC1 and PC2 respectively corresponded to $41.4 \%$ and $22.4 \%$ of the overall variation. Significant differences among the five growth stages were detected during this analysis, with bacterial communities during the elongation, booting, and grain filling stages being more similar than communities collected during the heading and ripening stages of growth. In addition, differences among treatment conditions were noted for samples collected at the elongation, booting, heading, and grain filling stages, whereas these samples appeared similar to one another at the ripening stage. 


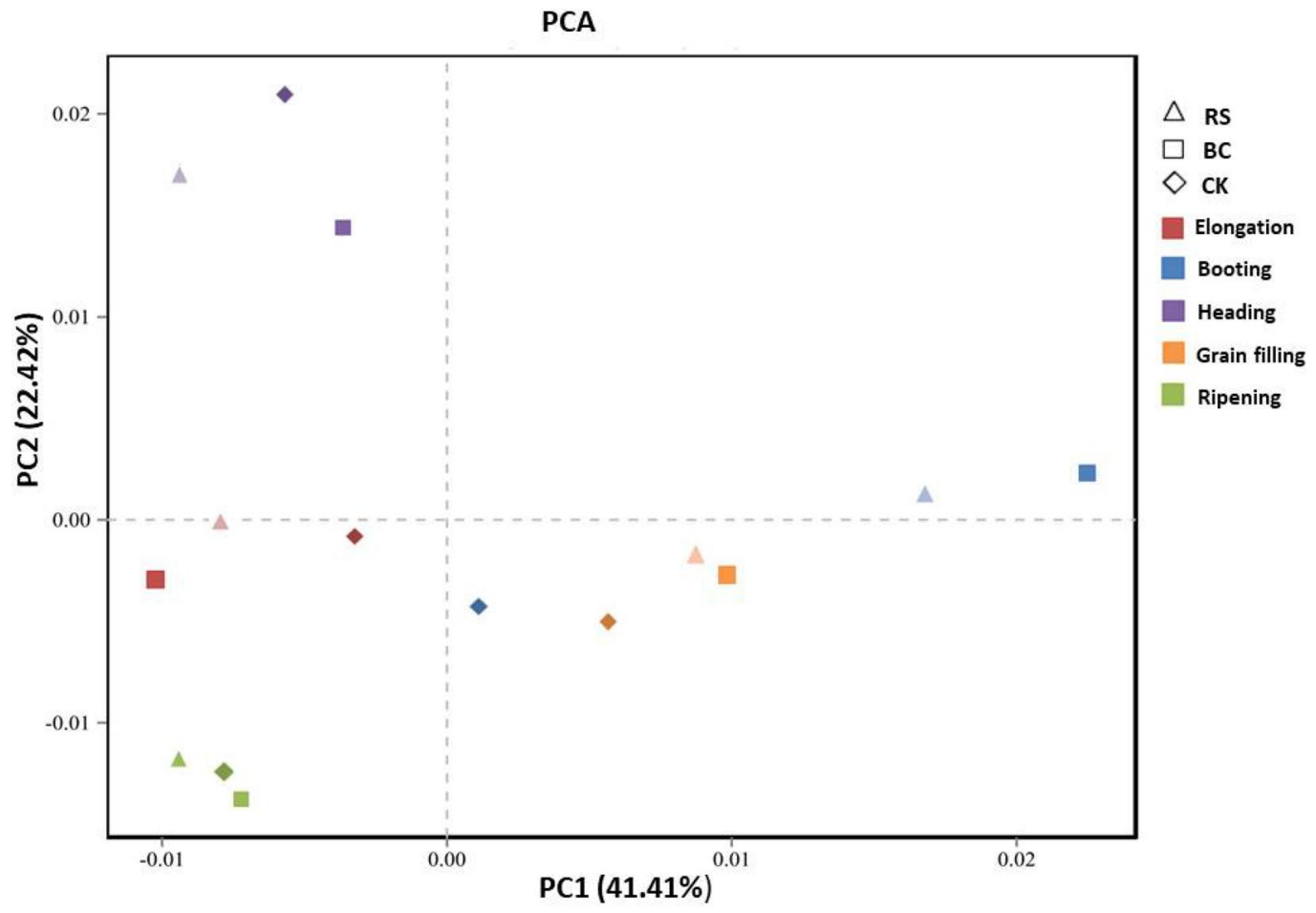

Figure 3. Principal component analysis of the bacterial genera in the rhizosphere soil samples. Treatments: $R S$ rice straw applied at $9000 \mathrm{~kg} \mathrm{ha}^{-1}, B C$ biochar applied at $3150 \mathrm{~kg} \mathrm{ha}^{-1}, C K$ soil without straw/biochar application.

RS and BC amendment alter the rhizosphere soil microbial community composition. Significant differences in rhizosphere $\alpha$-diversity values were detected among samples across the five rice growth stages $(\mathrm{P}=0.0017$, Kruskal-Wallis, Fig. 4). In elongation stage samples, $\alpha$-diversity values were higher for both RS and BC samples relative to CK controls, whereas RS and CK samples exhibited higher a-diversity values than BC samples at the booting stage of growth. At the heading stage, BC and RS samples exhibited reduced $\alpha$-diversity relative to CK samples, while BC samples presented with increased $\alpha$-diversity relative to RS and CK samples at the grain filling stage, while differences between the $\mathrm{BC}$ and $\mathrm{CK}$ samples were not significant. No significant differences were detected among groups when comparing samples collected at the ripening stage.

Relative root microbial abundance. Differences were found in the relative abundance of the top 30 genera among different treatments (Fig. 5). At the elongation stage, the dominant bacterial families in RS-treated samples were Gemmatimonadaceae and Sphingomonadaceae, while no families were clearly dominant in BC or CK samples. At the booting stage, Thiovulaceae was the dominant family in RS-treated samples of rhizosphere soil, while Thiovulaceae and uncultured bacterium-o-C0119 were dominant in BC-treated soil, and Unculturedbacterium-o-RBG-13-54-9 was dominant in the CK soil. At the heading stage, Burkholderiaceae and Clostridiaceae-1 were dominant in the RS-treated soil, while Uncultured-bacterium-o-Subgroup-7 and Micrococcaceae were dominant in the $\mathrm{CK}$ soil and no families were clearly dominant in BC-treated soil samples. No families were clearly dominant under any treatment conditions at the grain filling stage, while at the ripening stage Haliangiaceae was dominant in CK samples, whereas RS- and BC-treated soils did not exhibit any dominant bacterial families.

\section{Discussion}

Prior analyses have shown that crop rhizosphere microbial community composition and diversity vary depending on the types of plant residues applied to a given field, but these studies were conducted using upland crops or under greenhouse conditions $s^{14,18,19}$. In the present study, we focused on paddy soil rhizosphere microbes associated with rice plants, and assessed the Chaol and Shannon diversity indices for these communities following RS $\left(9000 \mathrm{~kg} \mathrm{ha}^{-1}\right), \mathrm{BC}\left(3150 \mathrm{~kg} \mathrm{ha}^{-1}\right)$, or control treatment during five stages of growth, enabling us to detect significant variations in soil microbiome diversity (Table 1). However, the OTUs in these samples did not differ significantly across all treatment conditions (Fig. 1), as these OTUs did not change in total quantity following RS or BC application relative to CK. RS and BC amendment only altered the relative abundance of these rootassociated microbes at the phyla level, consistent with the results previously published by Novak ${ }^{6}$ and $\mathrm{Zhao}^{20}$, who determined that the addition of $\mathrm{RS}$ and $\mathrm{BC}$ can increase available soil organic $\mathrm{C}$ and thereby provide a niche for soil microbial growth.

Chloroflexi, Proteobacteria, and Acidobacteria were the dominant phyla detected during all five stages of rice growth in the present study (Fig. 2), in line with prior research ${ }^{21-23}$. We found that Proteobacteria were the most abundant phyla in the RS-treated samples during the different stages of growth (Additional file: Figure S1, RS). 


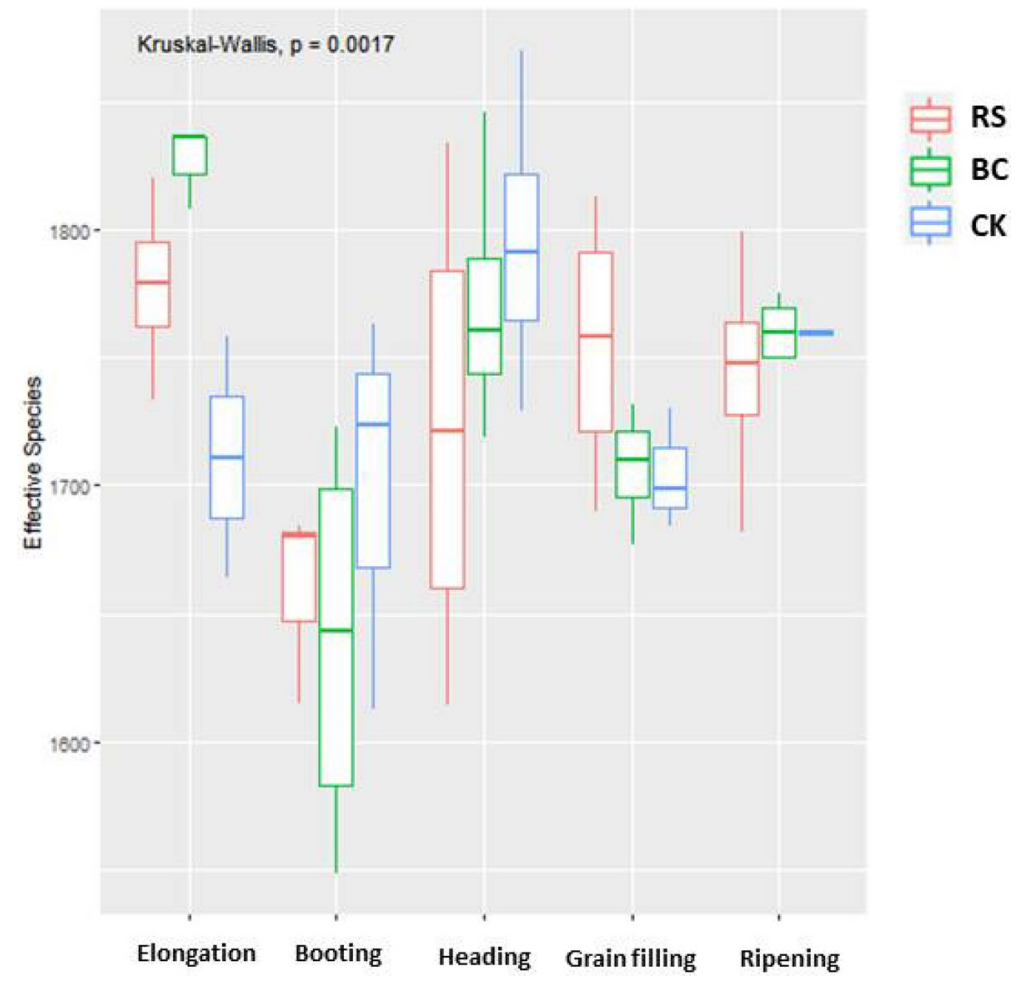

Figure 4. Alpha diversity indices for microbes in rhizosphere soil samples collected during different stages of rice growth. Treatments: $R S$ rice straw applied at $9000 \mathrm{~kg} \mathrm{ha}^{-1}, B C$ biochar applied at $3150 \mathrm{~kg} \mathrm{ha}^{-1}, C K$ soil without straw/biochar application.

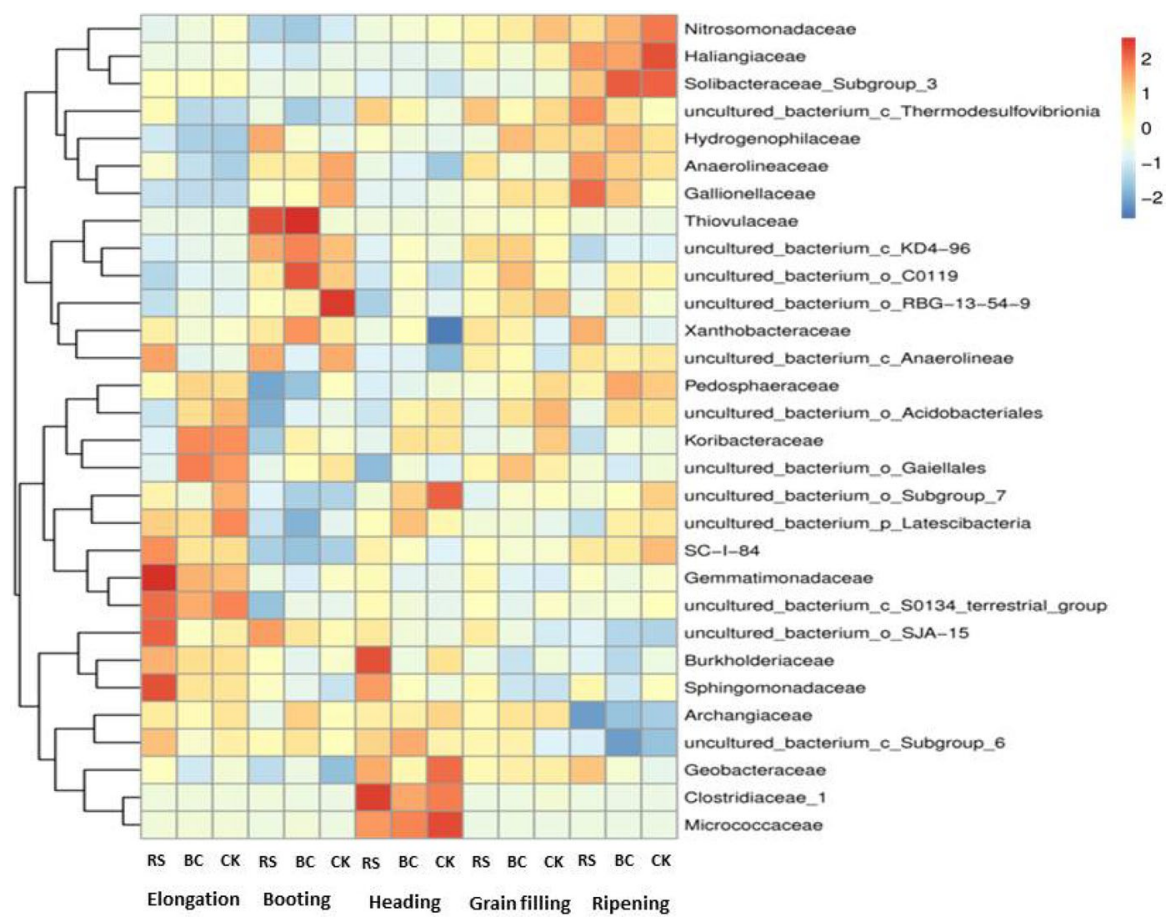

Figure 5. Relative abundance of top 30 genera in the treatment groups using $\mathrm{R}$ language ( $\mathrm{R} v 3.1 .1$, https://devel oper-platform.biocloud.net/\#/Microbial/taxonomySpecies/abundance_chart) to construct a species abundance heat map. Relative abundance is the correlation between species and treatments, with red representing a positive correlation and blue a negative correlation Treatments: $R S$ rice straw applied at $9000 \mathrm{~kg} \mathrm{ha}^{-1}, B C$ biochar applied at $3150 \mathrm{~kg} \mathrm{ha}^{-1}$,CK soil without straw/biochar application. 
This is consistent with reports that Proteobacteria are typically the dominant microbial phylum found in soil samples ${ }^{24,25}$. Proteobacteria can readily grow in nutrient-rich soil, and RS decomposition can facilitate significant nutrient release into soil ecosystem, supporting the growth of large numbers of these bacteria surrounding the root system. Chloroflexi was the most abundant phylum in the RS and BC samples across all analyzed rice growth stages (Additional file: Figure S1, BC, CK). Chloroflexi are anaerobic bacteria, and many microbes can grow in the rhizosphere under anaerobic conditions. The relative abundance of Chloroflexi in the analyzed samples varied with growth stage in an " $\mathrm{M}$ "-shaped pattern, potentially due to cultivation-related conditions. During the booting and grain filling stages of rice growth, paddy soil is saturated with water, whereas this water is not present during other growth stages. BC application increased relative rhizosphere soil Chloroflexi, Proteobacteria, and Acidobacteria abundance, likely because the $\mathrm{BC}$ provided high levels of $\mathrm{C}$ and an environmental niche compatible with the growth of these root-associated microbes owing to its stable, porous structure. Other groups have previously reported that $\mathrm{BC}$ addition can stimulate soil bacteria growth, with root-associated microbiota changing accordingly over the course of crop growth ${ }^{19,26}$, in line with our findings. High bacterial diversity and richness are typically associated with the proliferation of microbes capable of utilizing more diverse sources of carbon ${ }^{27}$. RS and BC application increased the relative Proteobacteria, Actinobacteria, and Gemmatimonadetes abundance in rhizosphere soil samples, consistent with the ability of these microbes to flourish in nutrient-rich settings ${ }^{28}$. Most eutrophication Proteobacteria in the RS- and BC-treated soil samples were present at higher levels than in CK samples at multiple growth stages (Fig. 2), suggesting that nutrient levels remained high in the amended rhizosphere soil throughout the growth period. Nitrospirae levels have been shown to rise following tillering and to remain at high levels during the rice reproductive stage ${ }^{29,30}$. Significant rises in Nitrospirae abundance in RS- and BC-treated rhizosphere soils (Fig. 2) can markedly enhance rice nitrate uptake. Overall, rhizosphere soil bacterial diversity and richness can be impacted by a range of variables including soil composition, cultivation methods, and management practices ${ }^{31}$. Analyzing the diversity of soil microbial communities can offer insight regarding the stability and fertility of the soil ecosystem, with Proteobacteria flourishing in nutrient-rich environments, while oligotrophic Actinobacteria tend to dominate in nutrient-poor environments wherein these slow-growing microbes can degrade more recalcitrant forms of $\mathrm{C}^{32}$. Consistent with the beneficial effects of RS application, we detected high levels of Proteobacteria in the RS-treated rhizosphere soil, indicating that C levels were abundant throughout the rice growth cycle, while the presence of Actinobacteria in BC-treated soils may indicate an environment that was somewhat more nutrient-poor.

Genus-level PCA results indicated that both RS and BC amendments significantly impacted soil microbial community composition from the elongation stage to the grain filling stage, although these differences were no longer evident at the ripening stage (Fig. 3). Prior reports have also shown that RS application can significantly alter soil microbial $\alpha$-diversity ${ }^{33}$. Indeed, we observed significant differences in rhizosphere $\alpha$-diversity measurements in samples from the RS and BC treatment groups relative to CK control samples (Fig. 4). Gemmatimonadaceae, Sphingomonadaceae, Thiovulaceae, Burkholderiaceae, and Clostridiaceae- 1 were the dominant families of microbes in RS-treated samples, whereas Thiovulaceae and uncultured-bacterium-o-C0119 were dominant in BC-treated samples (Fig. 5). RS application thus significantly altered the soil microbial community structure. Other studies have shown that levels and qualities of compounds released from crop straw can contribute to consequent changes in soil microbial communities ${ }^{11}$.

Straw and biochar harbor large quantities of $\mathrm{C}$, which serves as the main energy source for soil microorganisms ${ }^{39}$. Chloroflexi, Acidobacteria, Actinobacteria, and Verrucomicrobia levels were higher in BC samples relative to RS samples (Fig. 2). Chloroflexi are anaerobic bacteria, and the anaerobic conditions that arise during rice growth normalize and regulate soil bacterial community composition based on their oxygen requirements, resulting in stable bacterial communities in paddy soils ${ }^{40}$. Acidobacteria are acidophilic bacteria that play important roles in ecosystems, being primarily responsible for the decomposition of plant residue in the soil. Relative to straw application, BC input was associated with the increased abundance and diversity of these phyla under the water layer, potentially because the $\mathrm{pH}$ value of the soil solution may be changed due to alkalinity of the $\mathrm{BC}$, and maybe because these bacteria are able to inhabit the microporous structure of the BC. $\mathrm{BC}$ application may also alter other environmental factors including soil moisture, temperature, and $\mathrm{pH}$.

BC amendment was associated with a significant decrease in Proteobacteria, Gemmatimonadetes, Nitrospirae, and Firmicutes abundance relative to CK treatment during all growth stages in the present study (Fig. 2). Gemmatimonas were an abundant bacterial phyla associated with the RS treatment, potentially because Gemmatimonas use the RS as the sole source of available $\mathrm{C}^{41}$. Indeed, Gemmatimonas have been shown to reduce the metabolic products of cellulose $\mathrm{e}^{41}$, thereby indirectly facilitating cellulose degradation. Nitrospirae species play pivotal roles in nitrification by oxidizing nitrite to nitrate ${ }^{29}$. The enrichment of Nitrospirae may be due to root environmental changes during rice growth such as $\mathrm{pH}$ changes or root exudates. It is also possible that these microbes were actively recruited by rice to facilitate nitrate assimilation, potentially providing advantages with respect to nutrient uptake for rice cultivars during the elongating and booting stages. In this study, the relative abundance of Proteobacteria and Verrucomicrobia species was related to soil nutrient availability. We thus speculated that RS treatment may stimulate the relative proliferation of copiotrophic bacteria owing to the increased soil nutrient availability associated with RS decomposition.

At present, the application of RS to fields is the most common amendment method. In addition to contain large quantities of nutrients and trace elements, crop straw application can also increase the levels of soil organic matter, improve soil fertility, and significantly improve crop yields ${ }^{42}$. However, direct straw application is associated with issues including low temperatures, slow decomposition, and greenhouse gas emissions in northeast $\mathrm{China}^{43}$. BC is applied to fields in an innovative form of straw application. BC primarily affects the structure and function of microbial communities by maintaining carbon stability, improving soil quality, enhancing water retention, and changing $\mathrm{pH}^{44}$. 


\section{Conclusion}

Our results showed that the applications of RS and BC for rice production did not increase the number of observed OTUs, type of dominant phylum, family or genera variety in the rhizosphere soil bacterial community. On the other hand, RS and BC addition improved rhizosphere soil bacterial diversity and richness in phyla (Chloroflexi, Proteobacteria, and Acidobacteria) and clearly impacted the distribution of dominant family such as Gemmatimonadaceae, Sphingomonadaceae, Thiovulaceae, Thiovulaceae and uncultured-bacterium-o-C0119. Principal component analysis showed that the rhizosphere soil bacteria community differed significantly among the RS, BC and CK at all five stages of rice growth. RS and BC amendments have positive effects on soil fertility and productivity, helping farmers to reduce costs associated with straw disposal while increasing grain yields and improving production efficiency. Such amendment may also help improve soil productivity and sustainability in intensive agricultural systems. In comparison with synthetic fertilizers alone, the application of the RS and BC can improve soil microbial diversity and richness for rice production in Northeast China.

\section{Materials and methods}

Experimental design. The present study was conducted at Liaoning Rice Research Institute $\left(41^{\circ} 47^{\prime} \mathrm{N}\right.$, $123^{\circ} 34^{\prime}$ E, altitude: $40.5 \mathrm{~m}$ ) in Shenyang, Liaoning Province, China. Field experiments were initiated in 2019 to assess the impact of RS and $\mathrm{BC}$ amendment on soil bacterial community structure and biological traits. The soil at the study site is of a clayey loam type. At the start of the experiment, the soil exhibited a pH $\left(\mathrm{H}_{2} \mathrm{O}\right)$ of $5.2,19.2 \mathrm{~g} \mathrm{~kg}^{-1}$ organic matter, $1.29 \mathrm{~g} \mathrm{~kg}^{-1}$ total $\mathrm{N}, 1.27 \mathrm{~g} \mathrm{~kg}^{-1}$ total $\mathrm{P}, 23.7 \mathrm{~g} \mathrm{~kg}^{-1}$ total $\mathrm{K}, 109.0 \mathrm{mg} \mathrm{kg}^{-1}$ available $\mathrm{N}, 24.5 \mathrm{mg} \mathrm{kg}^{-1}$ available $\mathrm{P}$, and $45.0 \mathrm{mg} \mathrm{kg}^{-1}$ available $\mathrm{K}$. The study site is located in a region with a semihumid temperate and monsoonal type climate, with annual average temperature and precipitation of $8.2^{\circ} \mathrm{C}$ and $550 \mathrm{~mm}$, respectively.

The field experiment was designed with three treatments and four replicates per treatment in a completely randomized design, with each plot being $360 \mathrm{~m}^{2}$ in area. The treatments were as follows:

1. Rice straw (RS) was applied at $9000 \mathrm{~kg} \mathrm{ha}^{-1}$. RS was prepared by chopping dried straw and passing it through a $10 \mathrm{~mm}$ sieve. The resultant RS contained respective $\mathrm{C}$ and $\mathrm{N}$ levels of $354.8 \mathrm{~g} \mathrm{~kg}^{-1}$ and $6.8 \mathrm{~g} \mathrm{~kg}^{-1}$.

2. Biochar (BC) was applied at $3150 \mathrm{~kg} \mathrm{ha}^{-1}$. $\mathrm{BC}$ was prepared by subjecting RS to pyrolysis for $1 \mathrm{~h}$ under lowoxygen conditions at $450{ }^{\circ} \mathrm{C}$ (Jin and Fu Agriculture Development Co., Ltd), as this is comparable to the commercial process used during traditional furnace-based corn cob carbonization (Chinese patent ZL 2007 10086505.4). Using this approach, roughly $35 \%$ of the RS was converted into BC in the form of granular particles that were $2 \mathrm{~mm}$ in diameter. The resultant $\mathrm{BC}$ contained $660.4 \mathrm{~g} \mathrm{~kg}^{-1}$ of C and $7.9 \mathrm{~g} \mathrm{~kg}^{-1} \mathrm{of} \mathrm{N}$, and exhibited a $\mathrm{pH}$ of $8.6\left(1: 2.5 \mathrm{H}_{2} \mathrm{O}\right)$.

3. Control (CK) treatment without any soil amendment.

RS and BC were applied to experimental sites prior to rice transplanting in April 2019. These materials were incorporated into the field by hand using a rake, after which all plots were mechanically tilled to a uniform $0.15 \mathrm{~m}$ depth. The soil was additionally amended with calcium superphosphate and potassium chloride $(615 \mathrm{~kg}$ $\mathrm{P}_{2} \mathrm{O}_{5} \mathrm{ha}^{-1}$ and $\left.200 \mathrm{~kg} \mathrm{~K}_{2} \mathrm{O} \mathrm{ha}^{-1}\right)$, with additional urea being applied at the mid-tillering $\left(270 \mathrm{~kg} \mathrm{ha}^{-1}\right)$ and panicle initiation $\left(270 \mathrm{~kg} \mathrm{ha}^{-1}\right)$ stages of rice growth. $\mathrm{P}$ and $\mathrm{K}$ fertilizers were evenly applied as basal fertilizers on the soil surface and were promptly incorporated in the plow layer $(0-10 \mathrm{~cm})$ using a hand rake prior to transplanting.

The rice cultivar 'Liaoxing 21' was cultivated in 2019. The variety was widely used in a large northern part of area in Liaoning Province, and conventionally grown by local farmers. Rice seeds were sown in a nursery bed on April 19, 2019. Seedlings were transplanted to the paddy fields on May 26, 2019 and then harvested on October 20, 2019. Water levels, weed growth, insects, and diseases were managed as appropriate to prevent yield losses.

Rhizosphere soil collection. Soil samples were collected at the elongation, booting, heading, grain filling, and ripening stages of rice growth. At these times, plants and soil samples were collected from each plot, and five soil cores were pooled together to yield a composite sample. Three replicate samples were collected per treatment. Roots were removed from the soil and shaken to remove excessive soil until $\sim 1 \mathrm{~mm}$ of soil remained attached. This remaining soil was then obtained by placing roots in a flask containing $50 \mathrm{~mL}$ of sterile PBS. The solution was then vigorously stirred with sterile forceps to detach the rhizosphere soil, which was transferred to a $50 \mathrm{~mL}$ tube and frozen at $-80^{\circ} \mathrm{C}$ prior to DNA extraction.

$16 \mathrm{~S}$ rDNA sequencing and analysis. DNA was isolated from $0.5 \mathrm{~g}$ of soil with a Fast DNA SPIN Kit for Soil (Q-BIOgene, CA, USA) based on provided directions. DNA quality and quantity were assessed using an automated microplate reader (SynergyHTX, Gene Company Limited). The bacterial 16SrRNA V3-V4 region was then amplified with the 338F (5'-ACTCCTACGGGAGGCAGCA-3') and 806R (5'-ACTCCTACG GGAGGCAGCA-3') primers. A thermocycler (ABI GeneAmp 9700) was used for PCR amplification (25 or 10 cycles), with each reaction containing a total $10 \mu \mathrm{l}$ or $20 \mu \mathrm{l}$ volume. Successful amplification was confirmed via $1 \%$ agarose gel electrophoresis. After electrophoretic quantification using the ImageJ program, PCR products of all samples were combined together based on quality ratio values at a 1:1 ratio. Samples were purified using an OMEGA DNA purification column, and were mixed at equimolar ratios prior to sequencing with an Illumina HiSeq PE150 instrument (Illumina, USA) by Biomarker Technologies Co, LTD. Raw sequencing data were then merged with $\mathrm{FLASH}^{34}$, mass filtered ${ }^{35}$, and chimeric sequences removed to yield high-quality tagged sequences $^{36}$. These sequences, in turn, were clustered at a $97 \%$ similarity level ${ }^{37}$. 
Statistical analyses. Mothur was used to calculate OTU richness (ACE, Chao1), Shannon, and Simpson diversity indices ${ }^{38}$. Significant differences in microbial community composition among samples were identified via a permutational multivariate analysis of variance (PERMANOVA) approach. Microsoft Excel 2010 was used to graph the resultant data. Mothur was additionally used to compute principal component coordinates, while $\mathrm{R}$ ( v 3.0.2) was used to construct a PCA analysis diagram. R language ( $\mathrm{R}$ v 3.1.1, https://developer-platform.biocl oud.net/\#/Microbial/taxonomySpecies/abundance_chart) was used to construct a species abundance heat map.

Ethical standards. This project and the experiments were conducted in strict compliance with the IUCN Policy Statement on Research Involving Species at Risk of Extinction and the Convention on the Trade in Endangered Species of Wild Fauna and Flora. In our study that all plant research was carried out in accordance with national, international or institutional guidelines.

Received: 24 March 2021; Accepted: 9 September 2021

Published online: 12 November 2021

\section{References}

1. Cao, G., Zhang, X. \& Zheng, F. Inventory of black carbon and organic carbon emissions from China. Atmos. Environ. 40, 6516-6527 (2006).

2. Li, Y. T. et al. Microbial biomass enzyme and mineralization activity in relation to soil organic $\mathrm{C} \mathrm{N}$ and $\mathrm{P}$ turnover influenced by acid metal stress. Soil Biol Biochem. 41, 969-977 (2009).

3. Bera, T. et al. Changes in soil biochemical indicators at different wheat growth stages under conservation based sustainable intensification agriculture practices of rice-wheat system. J. Integr. Agric. 17, 1871-1880 (2018).

4. Kaewpradit, W. et al. Regulating mineral $\mathrm{N}$ release and greenhouse gas emissions by mixing groundnut residues and rice straw under field conditions. Eur. J. Soil Sci. 59, 640-652 (2008).

5. IBI. Standardized product definition and product testing guidelines for biochar that is used in soil. IBI Biochar Standards (2012).

6. Novak, J. M., Cantrell, K. B., Watts, D. W., Busscher, W. J. \& Johnson, M. G. Designing relevant biochars as soil amendments using lignocellulosic-based and manure-based feed stocks. J. Soils Sediments 14, 330-343 (2014).

7. Steinbeiss, S., Gleixner, G. \& Antonietti, M. Effect of biochar amendment on soil carbon balance and soil microbial activity. Soil Biol Biochem. 41, 1301-1310 (2009).

8. Cayuela, M. L. et al. Biochar and denitrification in soils: When, how much and why does biochar reduce $\mathrm{N}_{2} \mathrm{O}$ emissions?. Sci. Rep. 3, $1732(2013)$.

9. Jia, R., Qu, Z., You, P. \& Qu, D. Effect of biochar on photosynthetic microorganism growth and iron cycling in paddy soil under different phosphate levels. Sci. Total Environ. 612, 223-230 (2017).

10. Lehmann, J. et al. Biochar effects on soil biota-A review. Soil Biol Biochem. 43, 1812-1836 (2011).

11. Marschner, P., Kandeler, E. \& Marschner, B. Structure and function of the soil microbial community in a long-term fertilizer experiment. Soil Biol. Biochem. 35, 453-461 (2003).

12. Berendsen, R. L., Pieterse, C. M. J. \& Bakker, P. A. H. M. The rhizosphere microbiome and plant health. Trends Plant Sci. 17, 478-486 (2012).

13. Lundberg, D. S. et al. Defining the core Arabidopsis thaliana root microbiome. Nature 488, 86-90 (2012).

14. Edwards, J. et al. Structure, variation, and assembly of the root-associated microbiomes of rice. PNAS 112, E911-E920 (2015).

15. Böhme, L., Langer, U. \& Böhme, F. Microbial biomass, enzyme activities and microbial community structure in two European long-term field experiments. Agric. Ecosyst. Environ. 109, 141-152 (2005).

16. Navarrete, A. A. et al. Multi-analytical approach reveals potential microbial indicators in soil for sugarcane model systems. PLoS One 10, e012976510 (2015).

17. Börjesson, G., Menichetti, L., Kirchmann, H. \& Kätterer, T. Soil microbial community structure affected by 53 years of nitrogen fertilization and different organic amendments. Biol. Fertil. Soils 48, 245-257 (2012).

18. Chaparro, J. M., Badri, D. V. \& Vivanco, J. M. Rhizosphere microbiome assemblage is affected by plant development. ISME J. 8, 790-803 (2014).

19. Dombrowski, N. et al. Root microbiota dynamics of perennial Arabis alpina are dependent on soil residence time but independent of flowering time. ISME J. 11, 43-55 (2017).

20. Zhao, X., Wang, J., Wang, S. \& Xing, G. Successive straw biochar application as a strategy to sequester carbon and improve fertility: A pot experiment with two rice/wheat rotations in paddy soil. Plant Soil 378, 279-294 (2014).

21. Sun, J., Zhang, Q., Zhou, J. \& Wei, Q. Pyrosequencing technology reveals the impact of different manure doses on the bacterial community in apple rhizosphere soil. Appl. Soil Ecol. 78, 28-36 (2014).

22. Franke-Whittle, I. H., Manici, L. M., Insam, H. \& Stres, B. Rhizosphere bacteria and fungi associated with plant growth in soils of three replanted apple orchards. Plant Soil 395, 317-333 (2015).

23. Fan, W. \& Wu, J. Short-term effects of returning granulated straw on soil microbial community and organic carbon fractions in dryland farming. J. Microbiol. 58, 657-667 (2020).

24. Faoro, H. et al. Influence of soil characteristics on the diversity of bacteria in the Southern Brazilian Atlantic Forest. Appl. Environ. Microbiol. 76, 4744-4749 (2010).

25. Yin, C. T. et al. Members of soil bacterial communities sensitive to tillage and crop rotation. Soil Biol. Biochem. 42, 2111-2118 (2010).

26. Bamminger, C., Marschner, B. \& Jüschke, E. An incubation study on the stability and biological effects of pyrogenic and hydrothermal biochar in two soils. Eur. J. Soil Sci. 65, 72-82 (2014).

27. Fierer, N., Jackson, J. A., Vilgalys, R. \& Jackson, R. B. Assessment of soil microbial community structure by use of taxon-specific quantitative PCR assays. Appl. Environ. Microbiol. 71, 4117-4120 (2005).

28. Cheng, J. et al. Effect of biochar on the bioavailability of difenoconazole and microbial community composition in a pesticidecontaminated soil. Appl. Soil Ecol. 121, 185-192 (2014).

29. Kowalchuk, G. A. \& Stephen, J. R. Ammonia-oxidizing bacteria: A model for molecular microbial ecology. Annu. Rev. Microbiol. 55, 485-529 (2001).

30. Zhang, J. Y. et al. Root microbiota shift in rice correlates with resident time in the field and developmental stage. Sci. China Life Sci. 61, 613-621 (2018).

31. Schreiter, S. et al. Effects of the soil type on the microbiome in the rhizosphere of field-grown lettuce. Front. Microbiol. 5, 144 (2014). 
32. Jones, R. T. et al. A comprehensive survey of soil acidobacterial diversity using pyrosequencing and clone library analyses. ISME J. 3, 442-453 (2009).

33. Breulmann, M. et al. Short-term bioavailability of carbon in soil organic matter fractions of different particle sizes and densities in grassland ecosystems. Sci. Total Environ. 497-498, 29-37 (2014).

34. Magoč, T. \& Salzberg, S. L. FLASH: Fast length adjustment of short reads to improve genome assemblies. Bioinformatics 27, 2957-2963 (2011).

35. Bolger, A. M., Lohse, M. \& Usadel, B. Trimmomatic: A flexible trimmer for Illumina sequence data. Bioinformatics https://doi.org/ 10.1093/bioinformatics/btu170 (2014).

36. Edgar, R. C., Haas, B. J., Clemente, J. C., Quince, C. \& Knight, R. UCHIME improves sensitivity and speed of chimera detection. Bioinformatics 27, 2194-2200 (2011).

37. Edgar Robert, C. UPARSE: Highly accurate OTU sequences from microbial amplicon reads. Nat. Methods 10, 996-998 (2013).

38. Schloss, P. D. et al. Introducing mothur: Open-source, platform-independent, community-supported software for describing and comparing microbial communities. Appl. Environ. Microbiol. 75, 7537-7541 (2009).

39. Mathew, R. P., Feng, Y., Githinji, L., Ankumah, R. \& Balkcom, K. S. Impact of No-tillage and conventional tillage on soil microbial communities. Appl. Environ. Soil Sci. 2012, 1-10 (2012).

40. Kikuchi, H., Watanabe, T., Jia, Z., Kimura, M. \& Asakawa, S. Molecular analyses reveal stability of bacterial communities in bulk soil of a Japanese paddy field: Estimation by denaturing gradient gel electrophoresis of $16 \mathrm{~S}$ rRNA genes amplified from DNA accompanied with RNA. Soil Sci. Plant Nutr. 53(4), 448-458 (2007).

41. Takaichi, S., Maoka, M., Takasaki, K. \& Hanada, S. Carotenoids of Gemmatimonas aurantiaca (Gemmatimonadetes): Identification of a novel carotenoid, deoxyoscillol 2-rhamnoside, and proposed biosynthetic pathway of oscillol 2, 29-dirhamnoside. Microbiology 156(3), 757-763 (2010).

42. Sun, R., Zhang, X.-X., Guo, X., Wang, D. \& Chu, H. Bacterial diversity in soils subjected to long-term chemical fertilization can be more stably maintained with the addition of livestock manure than wheat straw. Soil Biol Biochem. 88, 9-18 (2015).

43. Liu, X. et al. Biochar's effect on crop productivity and the dependence on experimental conditions-A meta-analysis of literature data. Plant Soil 373(12), 583-594 (2013).

44. Li, M., Liu, M., Li, Z. P., Jiang, C. Y. \& Wu, M. Soil N transformation and microbial community structure as affected by adding biochar to a paddy soil of subtropical China. J. Integr. Agric. 15, 201-219 (2016).

\section{Acknowledgements}

We gratefully acknowledge The Earmarked Fund for Key Research and Development Project of Liaoning Province (2020JH2/10200031); China Agriculture Research System (CARS-01-50); Project of "Xingliao Talents Program" of Liaoning Province (XLYC1802119); Major agricultural project (2019JH1/10200001-2) support.

\section{Author contributions}

Z.T. contributed significantly to the field experiment, data collection and analysis, and wrote the original manuscript; L.Z., N.H., D.G., H.G., Z.M., L.F., M.Z., H.W., C.W., performed the experiment; W.Z. helped perform the analysis with constructive discussions; and W.Z. contributed to the conception of the study.

\section{Competing interests}

The authors declare no competing interests.

\section{Additional information}

Supplementary Information The online version contains supplementary material available at https://doi.org/ 10.1038/s41598-021-99001-9.

Correspondence and requests for materials should be addressed to W.Z.

Reprints and permissions information is available at www.nature.com/reprints.

Publisher's note Springer Nature remains neutral with regard to jurisdictional claims in published maps and institutional affiliations.

Open Access This article is licensed under a Creative Commons Attribution 4.0 International License, which permits use, sharing, adaptation, distribution and reproduction in any medium or format, as long as you give appropriate credit to the original author(s) and the source, provide a link to the Creative Commons licence, and indicate if changes were made. The images or other third party material in this article are included in the article's Creative Commons licence, unless indicated otherwise in a credit line to the material. If material is not included in the article's Creative Commons licence and your intended use is not permitted by statutory regulation or exceeds the permitted use, you will need to obtain permission directly from the copyright holder. To view a copy of this licence, visit http://creativecommons.org/licenses/by/4.0/.

(c) The Author(s) 2021 